\title{
The Exponential Time Hypothesis and the Parameterized Clique Problem
}

\author{
Yijia Chen $^{1}$, Kord Eickmeyer ${ }^{2 \star}$, and Jörg Flum ${ }^{3}$ \\ 1 Department of Computer Science, Shanghai Jiaotong University \\ yijia.chen@cs.sjtu.edu.cn \\ 2 National Institute of Informatics, Tokyo \\ eickmeye@nii.ac.jp \\ 3 Mathematisches Institut, Albert-Ludwigs-Universität Freiburg \\ joerg.flum@math.uni-freiburg.de
}

\begin{abstract}
In parameterized complexity there are three natural definitions of fixed-parameter tractability called strongly uniform, weakly uniform and nonuniform fpt. Similarly, there are three notions of subexponential time, yielding three flavours of the exponential time hypothesis (ETH) stating that 3SAT is not solvable in subexponential time. It is known that ETH implies that $p$-CLIQUE is not fixed-parameter tractable if both are taken to be strongly uniform or both are taken to be uniform, and we extend this to the nonuniform case. We also show that even the containment of weakly uniform subexponential time in nonuniform subexponential time is strict. Furthermore, we deduce from nonuniform ETH that no single exponent $d$ allows for arbitrarily good fpt-approximations of clique.
\end{abstract}

\section{Introduction}

In parameterized complexity, FPT most commonly denotes the class of strongly uniformly fixed-parameter tractable problems, i.e., parameterized problems solvable in time $f(k) \cdot n^{O(1)}$ for some computable function $f$. Downey and Fellows also introduced the classes $\mathrm{FPT}_{\mathrm{uni}}$ and $\mathrm{FPT}_{\mathrm{nu}}$ of uniformly and nonuniformly fixed-parameter tractable problems, where one drops the condition that $f$ be computable or allows for different algorithms for each $k$, respectively. (We give detailed definitions in Section 2.) For example, $p$-Clique $\notin \mathrm{FPT}_{\text {nu }}$, where $p$ Clique denotes the parameterized clique problem, means that for all $d \in \mathbb{N}$ and sufficiently large fixed $k$ determining whether a graph $G$ contains a clique of size $k$ is not in DTIME $\left(n^{d}\right)$. The obvious inclusions between the classes FPT, $\mathrm{FPT}_{\text {uni }}$, and $\mathrm{FPT}_{\text {nu }}$ can be shown to be strict [1].

In classical complexity, the subexponential time classes

$$
\operatorname{DTIME}\left(2^{o^{\text {eff }}(n)}\right), \quad \operatorname{DTIME}\left(2^{o(n)}\right), \quad \text { and } \bigcap_{\varepsilon>0} \operatorname{DTIME}\left(2^{\varepsilon \cdot n}\right),
$$

\footnotetext{
* This work was supported by a fellowship of the second author within the FITProgramme of the German Academic Exchange Service (DAAD).
} 
have been considered. In particular, there are the corresponding versions of the exponential time hypothesis, namely the strongly uniform exponential time hypothesis $\mathrm{ETH}$, the uniform exponential time hypothesis $\mathrm{ETH}_{\text {uni }}$, and the nonuniform exponential time hypothesis $\mathrm{ETH}_{\mathrm{nu}}$, which are the statements that 3SAT is not in these respective classes, where $n$ denotes the number of variables of the input formula. ${ }^{4}$ By results due to Impagliazzo et al. [3] we know that these three statements are equivalent to the ones obtained by replacing $3 \mathrm{SAT}$ by the clique problem Clique; then, $n$ denotes the number of vertices of the corresponding graph.

Furthermore, it is known [4] that ETH implies $p$-CLIQUE $\notin \mathrm{FPT}$, where $p$ Clique denotes the parameterized clique problem. As pointed out, for example in [5], there is a correspondence between subexponential algorithms having a running time $2^{o(n)}$ and $\mathrm{FPT}_{\text {uni }}$ similar to that between running time $2^{\text {off }^{\text {eff }}(n)}$ and FPT. Therefore, it is not surprising that $\mathrm{ETH}_{\text {uni }}$ implies $p$-CLIQUE $\notin \mathrm{FPT}_{\text {uni }}$ (we include a proof in Section 4).

The first main result of this paper shows that $\mathrm{ETH}_{\text {nu }}$ implies that $p$-CLIQUE $\notin$ $\mathrm{FPT}_{\mathrm{nu}}$. So, putting the three results together, we see that:

(i) if ETH holds, then $p$-CLIQUE $\notin \mathrm{FPT}$;

(ii) if $\mathrm{ETH}_{\text {uni }}$ holds, then $p$-CLIQUE $\notin \mathrm{FPT}_{\text {uni }}$;

(iii) if $\mathrm{ETH}_{\mathrm{nu}}$ holds, then $p$-CLIQUE $\notin \mathrm{FPT}_{\mathrm{nu}}$.

One of the most important complexity classes of (apparently) intractable parameterized problems is the class $\mathrm{W}[1]$, the class of problems (strongly uniformly) fpt-reducible to $p$-CliQuE. By replacing (strongly uniformly) fpt-reducible by uniformly fpt-reducible and nonuniformly fpt-reducible, we get the classes $\mathrm{W}[1]_{\mathrm{uni}}$ and $\mathrm{W}[1]_{\mathrm{nu}}$, respectively. So, the previous results can be seen as providing some evidence that $\mathrm{FPT} \neq \mathrm{W}[1], \mathrm{FPT}_{\mathrm{uni}} \neq \mathrm{W}[1]_{\mathrm{uni}}$, and $\mathrm{FPT}_{\mathrm{nu}} \neq \mathrm{W}[1]_{\mathrm{nu}}$. Note that the strongest separation, $\mathrm{FPT}_{\mathrm{nu}} \neq \mathrm{W}[1]_{\mathrm{nu}}$, obtained in this paper under $\mathrm{ETH}_{\mathrm{nu}}$, plays a role in [6] (see the comment following Theorem 1.1 of that paper). In a recent paper [7] on the history of Parameterized Complexity Theory, Downey remarks that the question $\mathrm{FPT}_{\mathrm{nu}} \neq \mathrm{W}[1]_{\mathrm{nu}}$ is a central issue of the theory.

We get the implication (iii) by using a family of algorithms witnessing $p$-CLI$\mathrm{QUE} \in \mathrm{FPT}_{\text {nu }}$ to obtain an algorithm showing $p$-CLIQUE is in "FPT $\mathrm{Tni}_{\text {un }}$ for positive instances." Once we have this single algorithm for $p$-CLIQUE, we adapt the techniques we used in [8] to show (i), to get that the clique problem Clique is in $\bigcap_{\varepsilon>0} \operatorname{DTIME}\left(2^{\varepsilon \cdot n}\right)$, that is, the failure of $\mathrm{ETH}_{\mathrm{nu}}$.

The basic idea of parameterized approximability is explained in [9] as follows: "Suppose we have a problem that is hard to approximate. Can we at least approximate it efficiently for instances for which the optimum is small? The classical theory of inapproximability does not seem to help answering this question, because usually the hardness proofs require fairly large solutions." In [9] and [10] the framework of parameterized approximability was introduced. In particular, the concept of an fpt approximation algorithm with a given approximation ratio was coined and some (in)approximability results were proven.

\footnotetext{
${ }^{4}$ We should mention that in [2] a different statement is called nonuniform ETH.
} 
Our second main result is a nonapproximability result for $p$-CLIQUE: under the assumption $\mathrm{ETH}_{\mathrm{nu}}$, we show that for every $d \in \mathbb{N}$ there is a $\rho>1$ such that $p$-CLIQUE has no parameterized approximation algorithm with approximation ratio $\rho$ and running time $f(k) \cdot n^{d}$ for some function $f: \mathbb{N} \rightarrow \mathbb{N}$.

The content of the different sections is the following. We recall concepts and fix notation in Section 2. In Section 3, we derive a result on the clique problem used to obtain both main results (in Section 4 and Section 5). Then, in Section 6 , we show that the results relating the complexity of $p$-CLIQUE and the different variants of ETH are of a more general nature. Finally, in Section 7, we show that the second and third time class in (1) are distinct.

\section{Some Preliminaries}

Let $\mathbb{N}$ denote the positive natural numbers. If a function $f: \mathbb{N} \rightarrow \mathbb{N}$ is nondecreasing and unbounded, then $f^{-1}$ denotes the function $f^{-1}: \mathbb{N} \rightarrow \mathbb{N}$ with

$$
f^{-1}(n):= \begin{cases}\max \{i \in \mathbb{N} \mid f(i) \leq n\}, & \text { if } n \geq f(1) \\ 1, & \text { otherwise }\end{cases}
$$

Then, $f\left(f^{-1}(n)\right) \leq n$ for all $n \geq f(1)$ and the function $f^{-1}$ is nondecreasing and unbounded.

Let $f, g: \mathbb{N} \rightarrow \mathbb{N}$ be functions. Then $f \in o^{\text {eff }}(g)$ (often written as $f(n) \in$ $\left.o^{\text {eff }}(g(n))\right)$ if there is a computable function $h: \mathbb{N} \rightarrow \mathbb{N}$ such that for all $\ell \geq 1$ and $n \geq h(\ell)$, we have $f(n) \leq g(n) / \ell$.

We denote the alphabet $\{0,1\}$ by $\Sigma$. The length of a string $x \in \Sigma^{*}$ is denoted by $|x|$. We identify problems with subsets $Q$ of $\Sigma^{*}$. If $x \in Q$ we say that $x$ is a positive instance of the problem $Q$. Clearly, as done mostly, we present concrete problems in a verbal, hence uncodified form. For example, we introduce the problem CLIQUE in the form:

\section{Clique}

Instance: A graph $G$ and $k \in \mathbb{N}$.

Problem: Does $G$ have a clique of size $k$ ?

A graph $G$ is given by its vertex set $V(G)$ and its edge set $E(G)$. By $|G|$ we denote the length of a string naturally encoding $G$. The cardinality or size of a set $S$ is also denoted by $|S|$.

If $\mathbb{A}$ is an algorithm and $\mathbb{A}$ halts on input $x$, then we denote by $t_{\mathbb{A}}(x)$ the number of steps of $\mathbb{A}$ on input $x$; if $\mathbb{A}$ does not halt on $x$, then $t_{\mathbb{A}}(x):=\infty$.

We view parameterized problems as pairs $(Q, \kappa)$ consisting of a classical problem $Q \subseteq \Sigma^{*}$ and a parameterization $\kappa: \Sigma^{*} \rightarrow \mathbb{N}$, which is required to be polynomial time computable. We will present parameterized problems in the form we do for $p$-CLIQUE: 


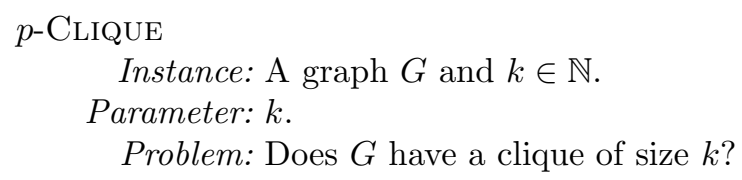

A parameterized problem $(Q, \kappa)$ is (strongly uniformly) fixed-parameter tractable or, in FPT, if there is an algorithm $\mathbb{A}$ deciding $Q$, a natural number $d$, and a computable function $f: \mathbb{N} \rightarrow \mathbb{N}$ such that $t_{\mathbb{A}}(x) \leq f(\kappa(x)) \cdot|x|^{d}$ for all $x \in \Sigma^{*}$.

If in this definition we do not require the computability of $f$, then $(Q, \kappa)$ is uniformly fixed-parameter tractable or, in $\mathrm{FPT}_{\text {uni }}$. Finally, $(Q, \kappa)$ is nonuniformly fixed-parameter tractable or, in $\mathrm{FPT}_{\mathrm{nu}}$, if there is a natural number $d$ and a function $f: \mathbb{N} \rightarrow \mathbb{N}$ such that for every $k \in \mathbb{N}$ there is an algorithm $\mathbb{A}_{k}$ deciding the set $\{x \in Q \mid \kappa(x)=k\}$ with $t_{\mathbb{A}_{k}}(x) \leq f(\kappa(x)) \cdot|x|^{d}$ for all $x \in \Sigma^{*}$.

In Section 6 we assume that the reader is familiar with the notion of (strongly uniform) fpt-reduction, with the classes of the W-hierarchy, and for $t, d \in \mathbb{N}$ with the weighted satisfiability problem $p$ - $\operatorname{WSAT}\left(\Gamma_{t, d}\right)$ (e.g., see [11]). We write $(Q, \kappa) \leq^{\mathrm{fpt}}\left(Q^{\prime}, \kappa^{\prime}\right)$ if there is an fpt-reduction from $(Q, \kappa)$ to $\left(Q^{\prime}, \kappa^{\prime}\right)$.

\section{Going from Nonuniform to Uniform on Positive Instances}

In this section we show how to get a single algorithm detecting cliques of size $k$ in time $f(k) \cdot|G|^{o(k)}$ on positive instances from the existence of such algorithms of running time $O\left(|G|^{e_{\ell}+k / \ell}\right)$ for each pair of natural numbers $k, \ell$. We now state this assumption formally; and we will later show it to be unlikely because it implies that $\mathrm{ETH}_{\mathrm{nu}}$ is not true.

Definition 1. We say that Clique satisfies $(*)$ if

for every $\ell \in \mathbb{N}$ there is an $e_{\ell} \in \mathbb{N}$ such that for every $k \in \mathbb{N}$ there is a constant $a_{\ell, k} \in \mathbb{N}$ and an algorithm $\mathbb{A}_{\ell, k}$ which on every graph $G$ which contains a clique of size $k$ outputs such a clique in time

$$
a_{\ell, k} \cdot|G|^{e_{\ell}+k / \ell} .
$$

The behaviour of $\mathbb{A}_{\ell, k}$ on graphs without a clique of size $k$ or on inputs not encoding graphs may be arbitrary.

By a standard self-reduction argument we have:

Lemma 2. If $p$-Clique $\in \mathrm{FPT}_{\mathrm{nu}}$, then Clique satisfies $(*)$.

We now use the algorithms in $(*)$ to obtain a single algorithm with a guaranteed running time on positive instances. 
Lemma 3. If Clique satisfies $(*)$, then there is an algorithm $\mathbb{A}$ deciding Clique and there is a function $f: \mathbb{N} \rightarrow \mathbb{N}$ such that

$$
t_{\AA}(G, k) \leq f(k) \cdot|G|^{o(k)}
$$

for every positive instance $(G, k)$ of Clique.

Proof. We let $\mathbb{C}$ be any algorithm which on input $(G, k)$ decides in time $|G|^{O(k)}$ whether $G$ contains a clique of size $k$, e.g., by brute force. Let $\left\{M_{1}, M_{2}, \ldots\right\}$ be any recursive enumeration of all Turing machines. By standard arguments we may assume that, given inputs $t, i$, and $x$, we can simulate $t$ steps of machine $M_{i}$ on input $x$ in time polynomial in $i, t$ and $|x|$.

We define the algorithm $\mathbb{A}$ as follows:

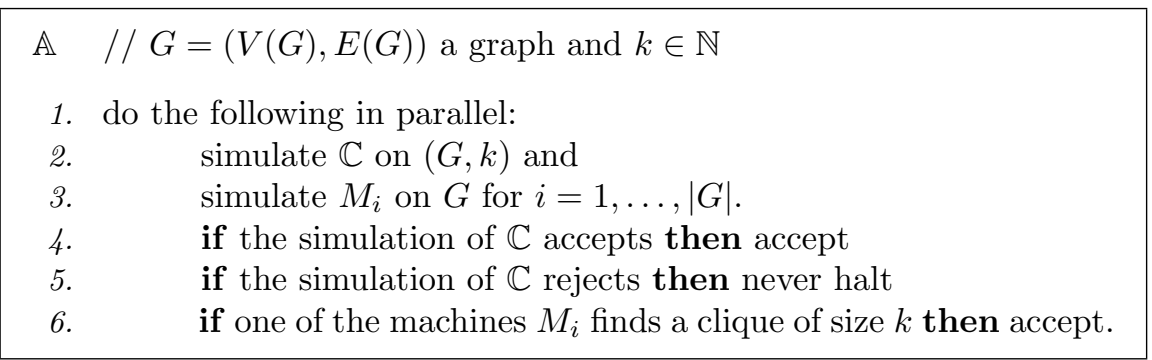

Obviously, this algorithm will accept an input $(G, k)$ if and only if $G$ contains a clique of size $k$. We now turn to the claimed running time. Let $\ell \geq 1$, and let $e_{\ell}$ be the corresponding constant from assumption $(*)$. For $k>\ell(\ell+1) e_{\ell}$, the running time of $\mathbb{A}_{\ell, k}$ is bounded by

$$
a_{\ell, k} \cdot|G|^{\frac{k}{\ell-1}},
$$

and for all but finitely many instances $G$, the algorithm $\mathbb{A}_{\ell, k}$ will be among the ones simulated by $\mathbb{A}$. For such instances $G$, the running time of $\mathbb{A}$ is bounded by

$$
\begin{aligned}
& ((\# \text { machines to be simulated in parallel }) \cdot(\# \text { of steps }) \cdot|G|)^{O(1)}, \\
\leq & \left(|G| \cdot a_{\ell, k} \cdot|G|^{\frac{k}{\ell-1}} \cdot|G|\right)^{O(1)} \\
\leq & c_{k} \cdot|G|^{\frac{d \cdot(k+1)}{\ell-1}}
\end{aligned}
$$

for suitable constants $c_{k}$ and $d$, the latter one not depending on $\ell, k$ or $G$.

\section{$4 \mathrm{ETH}_{\mathrm{nu}}$ and the Complexity of $p$-Clique}

In this section we show our first main result, namely:

Theorem 4. If $\mathrm{ETH}_{\mathrm{nu}}$ holds, then $p$-CLIQUE $\notin \mathrm{FPT}_{\mathrm{nu}}$. 
To obtain this result we prove the following chain of implications

$$
\text { (a) } \Rightarrow(\mathrm{b})_{\mathrm{pos}} \Rightarrow(\mathrm{c})_{\mathrm{pos}} \Rightarrow(\mathrm{d}) \text {, }
$$

where

(a) $p$-Clique $\in \mathrm{FPT}_{\mathrm{nu}}$;

(b) pos There is an algorithm $\mathbb{A}$ deciding CLIQUe such that for all positive instances $(G, k)$ of Clique and some function $f: \mathbb{N} \rightarrow \mathbb{N}$ we have

$$
t_{\mathbb{A}}(G, k) \leq f(k) \cdot|G|^{o(k)} .
$$

(c) pos There is an algorithm $\mathbb{A}$ deciding Clique such that for all positive instances $(G, k)$ of Clique, where $G$ has vertex set $V(G)$, we have

$$
t_{\mathbb{A}}(G, k) \leq 2^{o(|V(G)|)} .
$$

(d) $\mathrm{ETH}_{\text {nu }}$ does not hold.

Note that $\neg(\mathrm{d}) \Rightarrow \neg(\mathrm{a})$ is the claim of Theorem 4 .

The implication (a) $\Rightarrow(\mathrm{b})_{\text {pos }}$ was shown in the previous section (Lemma 2 and Lemma 3). We turn to the implication $(\mathrm{b})_{\text {pos }} \Rightarrow(\mathrm{c})_{\text {pos. }}$. Let (b) and (c) be the statements obtained from $(b)_{\text {pos }}$ and $(c)_{\text {pos }}$, respectively, by deleting the restriction to positive instances. Note that (c) is equivalent to the failure of $\mathrm{ETH}_{\mathrm{uni}}$. Furthermore, we let (b) eff be the statement (b) with the additional requirement that the function $f$ is computable and let $(\mathrm{c})_{\text {eff }}$ be the statement obtained from (c) by replacing $2^{o(|V(G)|)}$ by $2^{o^{\text {eff }}(|V(G)|)}$. Again note that $(\mathrm{c})_{\text {eff }}$ is equivalent to the failure of ETH.

Lemma 5. (1) (b) $)_{\mathrm{eff}}$ implies (c) $)_{\mathrm{eff}}$;

(2) (b) implies (c);

(3) (b) pos implies (c) pos.

Part (1) was shown as Theorem 27 in [8] (and previously in [4]). We argue similarly to get parts (2) and (3). In particular, we use the following lemma stated and proved in [8] as Lemma 28. Its proof uses the fact that a clique in a graph $G$ can be viewed as an "amalgamation of local cliques" of subgraphs of $G$.

Lemma 6. There is an algorithm $\mathbb{D}$ that assigns to every graph $G=(V, E)$ and $k, m \leq|V|$ in time polynomial in $|V| \cdot 2^{m}$ a graph $G^{\prime}=\left(V^{\prime}, E^{\prime}\right)$ with $\left|V^{\prime}\right| \leq|V|^{2} \cdot 2^{m}$ such that

$$
G \text { has a clique of size } k \Longleftrightarrow G^{\prime} \text { has a clique of size }\lceil|V| / m\rceil \text {. }
$$

Proof (of Lemma 5 (3)). The proof of Lemma 5 (2) is obtained by the obvious modification and is left to the reader.

Let the algorithm $\mathbb{D}$ be as in Lemma 6 . Assuming (b) pos there is an algorithm $\mathbb{A}$ deciding Clique such that for all positive instances $(G, k)$ of Clique we have $t_{\mathbb{A}}(G, k) \leq f(k) \cdot|G|^{o(k)}$ and hence,

$$
t_{\mathbb{A}}(G, k) \leq f(k) \cdot|V(G)|^{o(k)}
$$

for some $f: \mathbb{N} \rightarrow \mathbb{N}$. We consider the following algorithm deciding CuIQUE: 


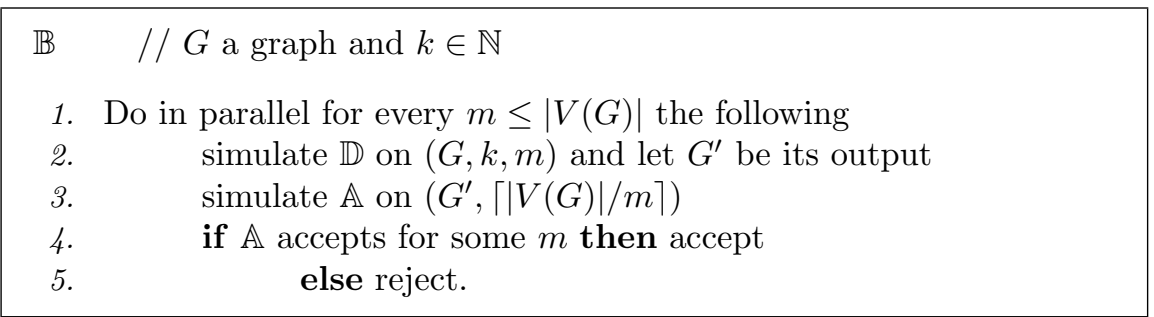

Let $(G, k)$ be a positive instance of CLIQUE and $n:=|V(G)|$. Without loss of generality, we can assume that $f$ is nondecreasing and unbounded. For

$$
m:=\max \left\{\left\lceil\frac{n}{f^{-1}(n)}\right\rceil,\lceil\log n\rceil\right\}
$$

we have $m \geq \log n$ and $m \in o(n)$ and, by (3),

$$
t_{\mathbb{A}}\left(G^{\prime},\lceil|V(G)| / m\rceil\right) \leq f(\lceil n / m\rceil) \cdot\left(n^{2} \cdot 2^{m}\right)^{o(n / m)}=2^{o(n)} .
$$

Thus, the running time for Line 2 to Line 5 is bounded by $2^{o(n)}$. Therefore

$$
t_{\mathbb{B}}(G, k) \leq O\left(n \cdot 2^{o(n)}\right) \leq 2^{o(n)} .
$$

We already remarked that (c) is equivalent to the failure of $\mathrm{ETH}_{\mathrm{uni}}$. Thus, part (2) of the previous lemma yields:

Corollary 7. If $\mathrm{ETH}_{\mathrm{uni}}$, then $p$-CLIQUE $\notin \mathrm{FPT}_{\mathrm{uni}}$.

Proof of $(\mathrm{c})_{\text {pos }} \Rightarrow(\mathrm{d})$ : For every $\epsilon>0$ there is an $n_{0}$ such that for graphs with $|V(G)|>n_{0}$ the running time of the algorithm asserted by (c) pos is bounded by $2^{\epsilon|V(G)|}$ on positive instances. For graphs with at least $n_{0}$ vertices we let the algorithm run for at most this many steps and reject if it does not hold within this time bound. For smaller graphs we use brute force.

For later purposes we remark:

Corollary 8. If Clique satisfies $(*)$, then $\mathrm{ETH}_{\mathrm{nu}}$ does not hold.

Proof. If Clique satisfies $(*)$, then $(\mathrm{b})_{\text {pos }}$ holds by Lemma 3. We have shown that (b) pos implies (d), thus, $\mathrm{ETH}_{\text {nu }}$ does not hold.

\section{$5 \mathrm{ETH}_{\text {nu }}$ and the Parameterized Approximability of p-Clique.}

Let $\rho>1$ be a real number. As in [9], we say that an algorithm $\mathbb{A}$ is an $f p t_{\text {uni }}$ parameterized approximation algorithm for $p$-CLIQUE with approximation ratio $\rho$ if

(i) $t_{\mathbb{A}}(G, k) \leq f(k) \cdot|V(G)|^{O(1)}$ for all instances $(G, k)$ of $p$-CLIQUE and some function $f: \mathbb{N} \rightarrow \mathbb{N}$ 
(ii) for all positive instances $(G, k)$ of $p$-Clique the algorithm $\mathbb{A}$ outputs a clique of size at least $k / \rho$; otherwise, the output of $\mathbb{A}$ can be arbitrary.

If $d \in \mathbb{N}$ and we get $t_{\mathbb{A}}(G, k) \leq f(k) \cdot|V(G)|^{d}$ in (i), then we say that $\mathbb{A}$ is an fpt $_{\text {uni }}$ parameterized approximation algorithm for $p$-CLIQUE with approximation ratio $\rho$ and exponent $d$.

Now we can state the main result of this section:

Theorem 9. If $\mathrm{ETH}_{\mathrm{nu}}$ holds, then for every $d \in \mathbb{N}$ there is a $\rho>1$ such that p-Clique has no fpt ${ }_{\text {uni }}$ parameterized approximation algorithm with approximation ratio $\rho$ and exponent $d$.

The key observation which, together with Corollary 8, will yield this theorem is contained in the following lemma.

Lemma 10. Assume that $p$-CLIQUE has an fpt $t_{\text {uni }}$ parameterized approximation algorithm with approximation ratio $\rho>1$ and exponent $d \geq 2$. Then, for every rational number $r$ with $0<r \leq \frac{1}{\log \rho}$, there is an algorithm $\mathbb{B}$ deciding CLIQUE such that for some function $g: \mathbb{N} \rightarrow \mathbb{N}$ and every instance $(G, k)$ of CLIQUE

$$
t_{\mathbb{B}}(G, k) \leq g(k) \cdot|V(G)|^{r+2+d \cdot\lceil k / r\rceil} .
$$

Proof. The main idea is as follows: we assume the existence of an fpt uni $_{\text {param- }}$ eterized approximation algorithm $\mathbb{A}$ for $p$-Clique. Given an instance $(G, k)$ of Clique we stretch it by passing to an equivalent "product instance" $\left(G^{\prime}, k^{\prime}\right)$. By applying $\mathbb{A}$ to $\left(G^{\prime}, k^{\prime}\right)$ we can decide whether $(G, k) \in$ Clique.

For a graph $G=(V, E)$ we let $\omega(G)$ be the size of a maximum clique in $G$. Furthermore, for every $m \in \mathbb{N}$ with $m \geq 1$ we denote by $G^{m}$ the graph $\left(V\left(G^{m}\right), E\left(G^{m}\right)\right)$, where

$$
\begin{aligned}
V\left(G^{m}\right):= & V^{m}=\left\{\left(v_{1}, \ldots, v_{m}\right) \mid v_{1}, \ldots, v_{m} \in V\right\} \\
E\left(G^{m}\right):= & \left\{\left\{\left(u_{1}, \ldots, u_{m}\right),\left(v_{1}, \ldots, v_{m}\right)\right\} \mid\left\{u_{1}, \ldots, u_{m}, v_{1}, \ldots, v_{m}\right\}\right. \\
& \text { is a clique in } \left.G \text { and }\left(u_{1}, \ldots, u_{m}\right) \neq\left(v_{1}, \ldots, v_{m}\right)\right\} .
\end{aligned}
$$

One easily verifies that

$$
\omega\left(G^{m}\right)=\omega(G)^{m} .
$$

Now we let $\mathbb{A}$ be an $\mathrm{fpt}_{\text {uni }}$ parameterized approximation algorithm for $p$-CLIQUE with approximation ratio $\rho>1$ and exponent $d \geq 2$, say, with running time bounded by $f(k) \cdot|V(G)|^{d}$. Let $r$ be a rational number with $0<r \leq \frac{1}{\log \rho}$. Then, $\rho \leq \sqrt[r]{2}$ and for every $k \in \mathbb{N}$ with $k \geq 2$ we get

$$
\left(\frac{k}{k-1}\right)^{\lceil k / r\rceil}>\rho
$$

We let $\mathbb{B}$ be the following algorithm: 


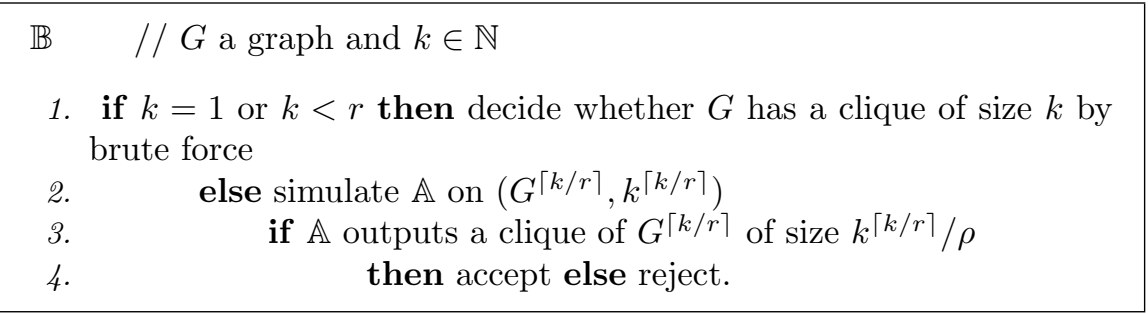

The algorithm $\mathbb{B}$ decides Clique: Clearly, the answer is correct if $k=1$ or $k<r$. So assume that $k \geq 2$ and $k \geq r$. If $G$ has no clique of size $k$, that is, $\omega(G) \leq k-1$, then, by $(4), \omega\left(G^{\lceil k / r\rceil}\right) \leq(k-1)^{\lceil k / r\rceil}$. By $(5)$,

$$
\frac{k^{\lceil k / r\rceil}}{\rho}>(k-1)^{\lceil k / r\rceil} ;
$$

thus, compare Line 3 and Line 4 , the algorithm $\mathbb{B}$ rejects $(G, k)$. If $\omega(G) \geq k$ and hence, $\omega\left(G^{\lceil k / r\rceil}\right) \geq k^{\lceil k / r\rceil}$, then the approximation algorithm $\mathbb{A}$ outputs a clique of $G^{\lceil k / r\rceil}$ of size $k^{\lceil k / r\rceil} / \rho$; thus $\mathbb{B}$ accepts $(G, k)$.

Moreover, on every instance $(G, k)$ with $G=(V, E)$ the running time of $\mathbb{B}$ is bounded by

$$
|V|^{r+2}+|V|^{2 \cdot\lceil k / r\rceil+2}+f\left(k^{\lceil k / r\rceil}\right) \cdot\left|V\left(G^{\lceil k / r\rceil}\right)\right|^{d} \leq g(k) \cdot|V|^{r+2+d \cdot\lceil k / r\rceil},
$$

for a suitable $g: \mathbb{N} \rightarrow \mathbb{N}$.

Setting $r:=1 / \log \rho$ in the previous lemma, we get:

Corollary 11. If there is an fpt $t_{\text {uni }}$ parameterized approximation algorithm for p-CLIQUE with approximation ratio $\rho \geq 1$ and exponent $d \geq 2$, then there exists $e \in \mathbb{N}$ and an algorithm $\mathbb{B}$ deciding CLIQUE with $t_{\mathbb{B}}(G, k) \leq \bar{g}(k) \cdot|V(G)|^{e+d \cdot k \cdot \log \rho}$

Proof of Theorem 9: By contradiction, assume that for some $d \geq 2$ and all $\rho>1$ the problem $p$-CLIQUE has an $\mathrm{fpt}_{\text {uni }}$ parameterized approximation algorithm with approximation ratio $\rho$ and exponent $d$.

If $\ell \in \mathbb{N}$, then $d \cdot \ell \leq \frac{1}{\log \rho}$ for suitable $\rho>1$. Thus, by Lemma 10, there is an algorithm $\mathbb{A}_{\ell}$ deciding CLIQUE such that for some $e_{\ell} \in \mathbb{N}$ and some function $g: \mathbb{N} \rightarrow \mathbb{N}$ and every instance $(G, k)$

$$
t_{\mathbb{A}_{\ell}}(G, k) \leq g(k) \cdot|V(G)|^{e_{\ell}+k / \ell} .
$$

Fix $k \in \mathbb{N}$. Then, again using the self-reducibility of CLIQUE, there is an algorithm $\mathbb{A}_{\ell, k}$ which on every graph $G$ outputs a clique of size $k$, if one exists, in time

$$
O\left(|G|^{e_{\ell}+1+k / \ell}\right) \text {. }
$$

Thus, Clique satisfies $(*)$ (the property introduced in Definition 1). Therefore, $\mathrm{ETH}_{\text {nu }}$ does not hold by Corollary 8 . 


\section{Some Extensions and Generalisations}

Some results of Section 3 and of Section 4 can be stated more succinctly and in a more general form in the framework of parameterized complexity theory. We do this in this section, at the same time getting some open questions.

The class $\mathrm{FPT}_{\text {nu }}$ is closed under fpt-reductions, that is,

$$
\text { if }(Q, \kappa) \leq^{\mathrm{fpt}}\left(Q^{\prime}, \kappa^{\prime}\right) \text { and }\left(Q^{\prime}, \kappa^{\prime}\right) \in \mathrm{FPT}_{\mathrm{nu}} \text {, then }(Q, \kappa) \in \mathrm{FPT}_{\mathrm{nu}} .
$$

Thus, for every W[1]-complete problem $(Q, \kappa)$ (complete under fpt-reductions), we have

$$
(Q, \kappa) \in \mathrm{FPT}_{\mathrm{nu}} \Longleftrightarrow \mathrm{W}[1] \subseteq \mathrm{FPT}_{\mathrm{nu}} .
$$

Denote by $\mathrm{FPT}_{\text {uni }}^{+}$the class of problems $(Q, \kappa)$ such that there is an algorithm deciding $Q$ and with running time $h(\kappa(x)) \cdot|x|^{O(1)}$ for $x \in Q$, that is, for positive instances $x$ of $Q$. The class $\mathrm{FPT}_{\mathrm{uni}}^{+}$is closed under fpt-reductions, too. So, again we have for every $\mathrm{W}[1]$-complete problem $(Q, \kappa)$,

$$
(Q, \kappa) \in \mathrm{FPT}_{\text {uni }}^{+} \Longleftrightarrow \mathrm{W}[1] \subseteq \mathrm{FPT}_{\mathrm{uni}}^{+}
$$

Corollary 12. For every $\mathrm{W}[1]$-complete problem $(Q, \kappa)$,

$$
(Q, \kappa) \in \mathrm{FPT}_{\mathrm{nu}} \text { implies }(Q, \kappa) \in \mathrm{FPT}_{\mathrm{uni}}^{+} .
$$

Proof. By Lemma 2 and Lemma 3, we know that the implication holds for the $\mathrm{W}[1]$-complete problem $p$-CLIQUE. Now, the claim follows by (7) and (8).

It is not clear whether the previous implication holds for all problems $(Q, \kappa) \in$ $\mathrm{W}[1]$ (and not only for the complete ones). Of course, it does if FPT $=\mathrm{W}[1]$. The proof of Lemma 3 makes essential use of a self-reducibility property of $p$ Clique. For $t, d \in \mathbb{N}$ the weighted satisfiability problem $p$-WSAT $\left(\Gamma_{t, d}\right)$ has this self-reducibility property, too. So, along the lines of Lemma 3, one gets (we leave the details to the reader):

Lemma 13. Let $t, d \in \mathbb{N}$. Then

$$
p-\operatorname{WSAT}\left(\Gamma_{t, d}\right) \in \mathrm{FPT}_{\text {nu }} \quad \text { implies } \quad p-\operatorname{WSAT}\left(\Gamma_{t, d}\right) \in \mathrm{FPT}_{\text {uni }}^{+} \text {. }
$$

And thus, we get the extension of Corollary 12 to all levels of the W-hierarchy:

Proposition 14. Let $t \in \mathbb{N}$. For every $\mathrm{W}[t]$-complete problem $(Q, \kappa)$,

$$
(Q, \kappa) \in \mathrm{FPT}_{\mathrm{nu}} \text { implies }(Q, \kappa) \in \mathrm{FPT}_{\mathrm{uni}}^{+} .
$$

After Theorem 4, we have considered two further properties of the clique problem there denoted by $(b)_{\text {pos }}$ and $(c)_{\text {pos }}$. One could also define these properties for arbitrary parameterized problems (even though, there are some subtle points as the terms $2^{o(|V(G)|)}$ and $2^{o(|G|)}$ may be distinct). More importantly, these properties are not closed under fpt-reductions. So somehow one has to check whether other implications of Section 4 survive problem by problem. We do that here for the most prominent $\mathrm{W}[2]$-complete problem, the parameterized dominating set problem $p$-DS: 


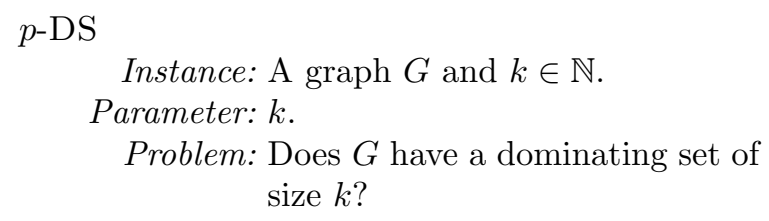

We denote by DS the underlying classical problem. In [8, Theorem 29] we have shown:

$$
\begin{aligned}
& \text { If } \mathrm{DS} \text { can be decided in time } f(k) \cdot|V(G)|^{\rho^{\text {eff }}(k)} \text { for some computable } \\
& f: \mathbb{N} \rightarrow \mathbb{N} \text {, then } \mathrm{DS} \text { can be decided in time } 2^{o^{\text {eff }}(|V(G)|)} \text {. }
\end{aligned}
$$

The reader should compare this result with the following one in the spirit of this paper.

Theorem 15. If there is an algorithm $\mathbb{A}$ deciding DS such that for all positive instances $(G, k)$ of DS we have

$$
t_{\mathbb{A}}(G, k) \leq f(k) \cdot|G|^{o(k)}
$$

for some function $f: \mathbb{N} \rightarrow \mathbb{N}$, then there is an algorithm $\mathbb{B}$ deciding DS such that for all positive instances $(G, k)$ of DS we have

$$
t_{\mathbb{B}}(G, k) \leq 2^{o(|V(G)|)} .
$$

The proof of the corresponding result for CLIQUE, namely the implication (b) $)_{\mathrm{pos}} \Rightarrow$ $(c)_{\text {pos }}$, was based on Lemma 6 which used the fact that a clique in a graph can be viewed as an "amalgamation of local cliques" of subgraphs. As dominating sets are not necessarily an "amalgamation of local dominating sets," in [8] we took a detour via the weighted satisfiability problem for propositional formulas in CNF. As an inspection of the exposition in [8] shows, it can be adapted to a proof of Theorem 15.

\section{$7 \quad$ An Example}

We believe that the three statements ETH, $\mathrm{ETH}_{\text {uni }}$, and $\mathrm{ETH}_{\mathrm{nu}}$ are true and hence equivalent. Here we consider the "underlying" complexity classes (see (1)). Clearly,

$$
\operatorname{DTIME}\left(2^{o^{\text {eff }}(n)}\right) \subseteq \operatorname{DTIME}\left(2^{o(n)}\right) \subseteq \bigcap_{\varepsilon>0} \operatorname{DTIME}\left(2^{\varepsilon \cdot n}\right)
$$

To the best of our knowledge it is open whether the first inclusion is strict. Here we show the strictness of the second inclusion in (9). We remark that in $[8$, Proposition 5] we proved that the first class, that is, the effective version of the second one, coincides with an effective version of the third class.

For $m \in \mathbb{N}$ let $1^{m}$ be the string in $\Sigma^{*}$ consisting of $m$ ones. Recall that $\Sigma=\{0,1\}$. For a Turing machine $\mathbb{M}$ we denote by enc $(\mathbb{M})$ a string in $\Sigma^{*}$ reasonably encoding the Turing machine $\mathbb{M}$. Furthermore, $|\mathbb{M}|$ denotes the length of enc $(\mathbb{M}),|\mathbb{M}|=|\operatorname{enc}(\mathbb{M})|$. 
Theorem 16. The problem

EXP-HALT

Instance: A Turing machine $\mathbb{M}, x \in \Sigma^{*}$, and $1^{m}$ with $m \in \mathbb{N}$.

Problem: Does $\mathbb{M}$ accept $x$ in time $2\lfloor m /(|\mathbb{M}|+|x|)\rfloor$ ?

is in $\bigcap_{\varepsilon>0}$ DTIME $\left(2^{\varepsilon \cdot n}\right) \backslash \operatorname{DTIME}\left(2^{o(n)}\right)$.

Due to space limitations we cannot present a proof in this extended abstract.

\section{References}

1. Downey, R., Fellows, M.: Fixed-parameter tractability and completeness iii: some structural aspects of the w hierarchy. In Ambos-Spies, K., Homer, S., Schöning, U., eds.: Complexity theory, New York, NY, USA, Cambridge University Press (1993) $191-225$

2. Ganian, R., Hlinený, P., Langer, A., Obdrzálek, J., Rossmanith, P., Sikdar, S.: Lower bounds on the complexity of $\mathrm{MSO}_{1}$ model-checking. In: Proc. STACS'12. (2012) 326-337

3. Impagliazzo, R., Paturi, R., Zane, F.: Which problems have strongly exponential complexity? Journal of Computer and System Sciences 63 (2001) 512-530

4. Chen, J., Huang, X., Kanj, I.A., Xia, G.: Linear fpt reductions and computational lower bounds. In: Proc. of STOC'04. (2004) 212-221

5. Flum, J., Grohe, M.: Parametrized complexity and subexponential time (column: Computational complexity). Bulletin of the EATCS 84 (2004) 71-100

6. Grohe, M.: The complexity of homomorphism and constraint satisfaction problems seen from the other side. J. ACM 54(1) (2007)

7. Downey, R.: The birth and early years of parameterized complexity. In: The Multivariate Algorithmic Revolution and Beyond. (2012) 17-38

8. Chen, Y., Flum, J.: On miniaturized problems in parameterized complexity theory. Theoretical Computer Science 351(3) (2006) 314-336

9. Chen, Y., Grohe, M., Grüber, M.: On parameterized approximability. In: 2nd International Workshop on Parameterized and Exact Computation. Number 4169 in LNCS, Springer-Verlag (2006) 109-120

10. Marx, D.: Parameterized complexity and approximation algorithms. The Computer Journal 51 (2008) 60-78

11. Flum, J., Grohe, M.: Parameterized Complexity Theory. Springer-Verlag, Berlin Heidelberg (2006) 